\title{
EL GÉNERO Clypeaster (ECHINOIDEA, CLYPEASTEROIDA) EN EL MIOCENO SUPERIOR DE VILLANUEVA DEL RÍO Y MINAS (SEVILLA, SO DE ESPAÑA)
}

\author{
Ildefonso BAJO CAMPOS
}

C/ Fuerteventura $n^{\circ} 2,41700$ Dos Hermanas

(Sevilla).E-mail: ildebajo@wanadoo.es

Bajo Campos, I. 2002. El género Clypeaster (Echinoidea, Clypeasteroida) en el Mioceno Superior de Villanueva del Río y Minas (Sevilla, SO de España). [The genus Clypeaster (Echinoidea, Clypeasteroida) from the Upper Miocene of Villanueva del Río y Minas (Seville, SW Spain).] Revista Española de Paleontología, 17(2), 177188. ISSN 0213-6937.

\begin{abstract}
Clypeaster specimens frequently appear in Oligo-Miocene shallow marine carbonate facies, and specially those of the Mediterranean realm (Boggild and Rose, 1985). In this paper, seven species of Clypeaster are described from a new locality of Late Miocene age: C. brevior (Seguenza), C. franchii (Checchia-Rispoli), C. cermanatii (Checchia-Rispoli), C. portentosus (Desmoulins), C. aichinoi (Checchia-Rispoli), C. tauricus (Desor) and Clypeaster sp. Five of them: C. franchii, C. cermanatii, C. aichinoi, C. tauricus and Clypeaster sp, are mentioned from the Iberian Peninsula for the first time, enlarging their paleogeographic distribution towards the Atlantic domain. Initially a shallow littoral environment characterized by turbulent waters was established containing large species. Later the environment changed to more quiet and deeper waters with a fine susbstrate in which lived smaller species.
\end{abstract}

Keywords: Clypeaster, Upper Miocene, systematic, SW Spain.

\section{RESUMEN}

Los ejemplares de las especies del género Clypeaster suelen aparecer con frecuencia en facies carbonatadas marinas someras de edad Cenozoica y en especial las del dominio Mediterráneo (Boggild y Rose, 1985). En este trabajo se describen siete especies de Clypeaster: C. brevior (Seguenza), C. franchii (Checchia-Rispoli), $C$. cermanatii (Checchia-Rispoli), C. portentosus (Desmoulins), C. aichinoi (Checchia-Rispoli), C. tauricus (Desor) y Clypeaster sp., procedentes de un nuevo yacimiento del Mioceno Superior y de las cuales cinco: $C$. franchii, $C$. cermanatii, C. aichinoi, C. tauricus y Clypeaster sp. se citan por primera vez en la Península Ibérica, ampliándose su distribución paleogeográfica hacia el dominio Atlántico. Inicialmente se estableció un ambiente litoral somero de poca profundidad y con aguas agitadas, en el que proliferaron taxones de gran tamaño. Posteriormente evolucionó hacia otro de aguas más tranquilas y profundas con substrato más fino que propició la sustitución de estas especies por otras de menor tamaño.

Palabras clave: Clypeaster, Mioceno Superior, sistemática, SO España.

\section{INTRODUCCIÓN Y ANTECEDENTES}

Las publicaciones sobre Clypeaster en España son escasas. Una de las primeras citas sobre su presencia se refiere a Clypeaster scutellatus (Serres, 1829, p. 157) en las "calcaire moellon de Barcelona". A partir de esta fecha las citas se multiplican (Cotteau, 1890; Mallada, 1892). En la primera mitad del siglo XX destaca el trabajo de Lambert (1928) en el que describe un total de doce especies, y cuatro décadas más tarde, Montenat y Roman (1970) realizan un estudio sobre los equinodermos neógenos de las provincias de Alicante y Murcia donde señalan la presencia de diecinueve especies del género Clypeaster en el Mioceno Superior.

La primera mención sobre la presencia de clypeastéridos en la zona se debe a Kilian (1892) en la que cita la presencia de Clypeaster altus (Klein) y Clypeaster pyramidalis (Seguenza). Así mismo, en la Hoja de Lora del Río (García et al., 1975) se cita la presencia de Clypeaster aff. marginatus, y Galán et al. (1993), en la Guía del Museo de Geología de la Universidad de Sevilla, citan la existencia de $C$. insignis procedente de Villanueva del Río y Minas. 


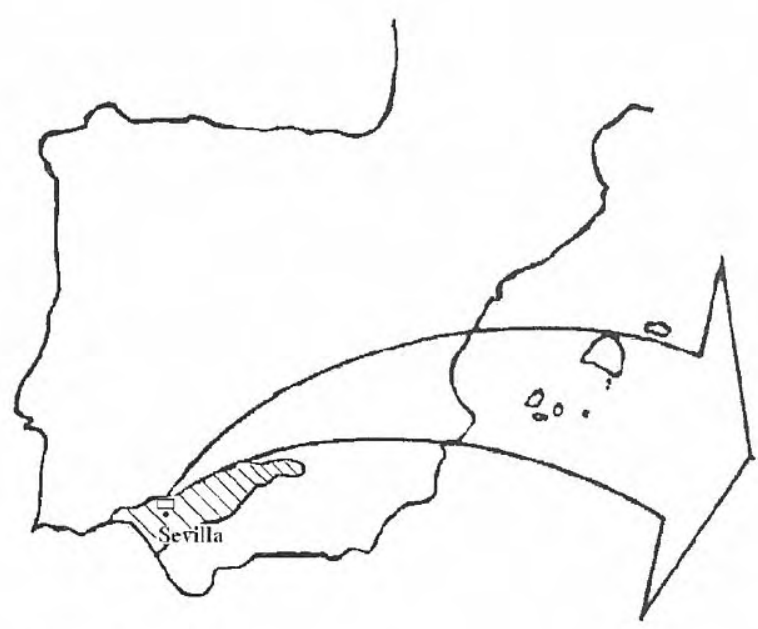

Cuenca del Guadalquivir
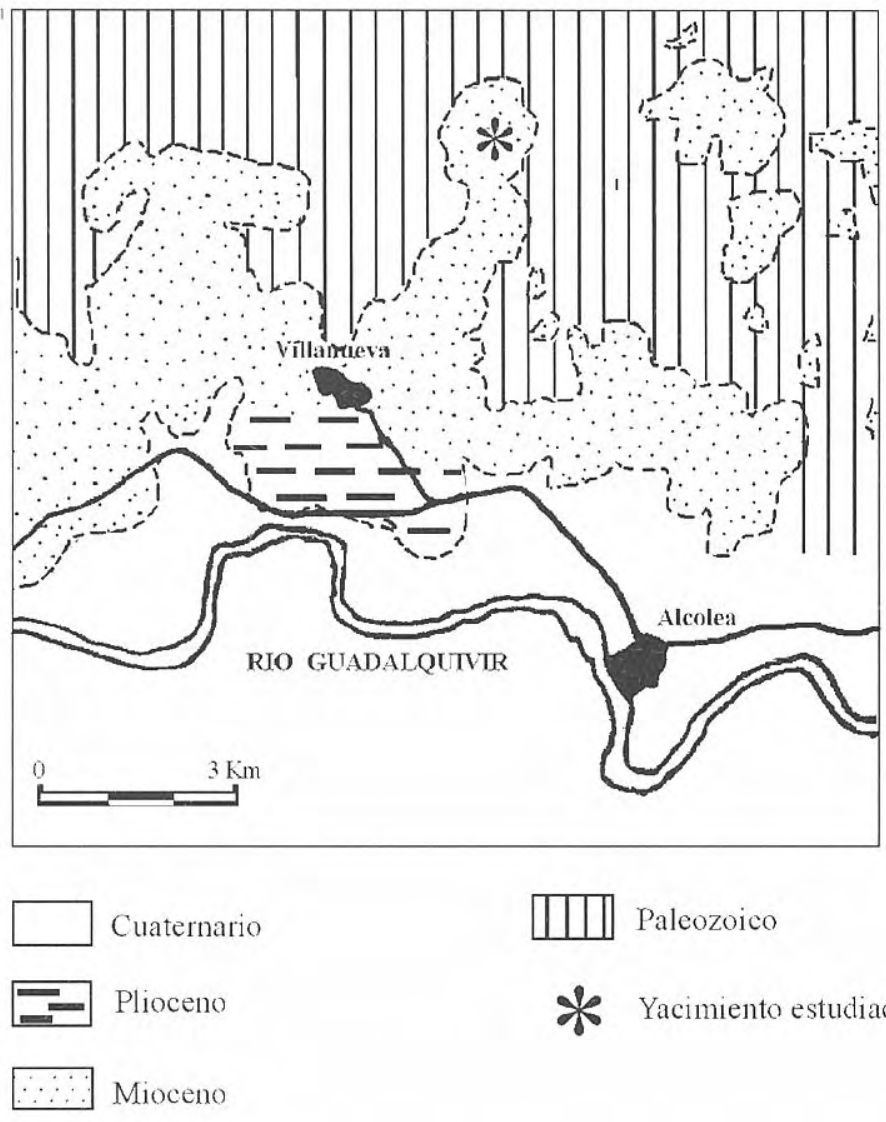

Cuaternario

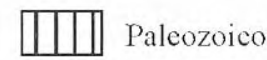

Plioceno

Mioceno

Figura 1. Situación geográfica y contexto geológico del yacimiento estudiado. Geographical location and geological context of the studied outcrop.

\section{CONTEXTO GEOLÓGICO Y LOCALIZACIÓN DEL YACIMIIENTO}

El yacimiento estudiado aflora unos $5 \mathrm{~km}$ al norte de Villanueva del Río y Minas (Sevilla), en una cantera que se explota para la extracción de arenas (Fig. 1). En este sector aflora el Mioceno Superior terminal (Sierro et al., 1990) .

La sucesión litoestratigráfica local se representa en la Fig. 2 . Consta de muro hacia techo de los siguientes tramos:

Tramo I: $4 \mathrm{~m}$ de arenas finas granocrecientes, con estratificación cruzada de gran escala y base curva, que contienen restos de malacofauna (bivalvos, gasterópodos, escafópodos) de pequeño tamaño (máximo $3 \mathrm{~cm}$ ) indeterminables. Aparecen hacia el techo de este tramo arenas intensamente bioturbadas por madrigueras de sección circular.

Tramo II : Banco de ostreidos (Crassostrea gryphoides Schlotheim) de unos dos metros de potencia, con matriz arenosa, presentando hacia la mitad de éste abundantes equínidos: Echinolampas sp., en posición de vida, aunque deformados por la presión litostática.

Tramo III: Sobre los materiales anteriores, se dispone de forma concordante un paquete de unos $8 \mathrm{~m}$ de espesor que está constituido en su parte inferior por arenas de grano medio, las cuales presentan esporádicamente dientes de seláceos (Isurus hastalis Agassiz), y de perciformes (Sparus sp.), abundantes restos de cetáceos y moldes de bivalvos (Pholadomya alpina Matheron). Hacia la mitad del tramo se intercala un nivel de conglomerados de espesor decimétrico, con moldes de bivalvos articulados (Isognomon sp., Venus sp., Glycimeris sp.), gasterópodos (Conus sp., Xenophora infundibulum Brocchi), ostreidos y restos leñosos con Teredolites ichnosp. indet. Justo por debajo de este conglomerado aparece el primer registro de Clypeaster portentosus. En la parte superior de este tramo, las arenas se hacen más finas, presentando niveles centimétricos constituidos por moldes de gasterópodos (Turritella sp.).

Tramo IV: Arenas bioclásticas de grano medio, con Heterostegina y estratificación masiva, de unos dos metros de potencia y granodecreciente, en el que aparecen restos de cetáceos y de gasterópodos (Scaphander lignaria Linné, Ficus sp., Conus sp.), bivalvos (Gigantopecten albinus Vontppner, Anomia ephippium Linné, Leporimetis lacunosa Chemnitz) y Clypeaster de gran tamaño con formas elevadas y bordes gruesos, normalmente en posición de máximo equilibrio mecánico (con la cara apical dispuesta hacia el techo y la oral paralela al plano de estratificación).

Tramo V: Lo forman unas arenas limosas de grano fino intensamente bioturbadas, con unos ocho metros de potencia, en las que aparecen bivalvos articulados (Flabellipecten solarium Lamarck, Chlamys multistriata Poli, Flabellipecten flaterculus Sowerby, Flabellipecten burdigalensis Lamarck) y abundantes equinodermos (Clypeaster sp., Clypeaster portentosus, este último muy escaso). Este tramo finaliza con un nivel cementado de arenas.

Tramo VI: Margas de color amarillento, con nódulos de carbonatos, de $1 \mathrm{~m}$ de potencia, sin macrofauna aparente, que se sitúa en paraconformidad sobre el tramo anterior. 


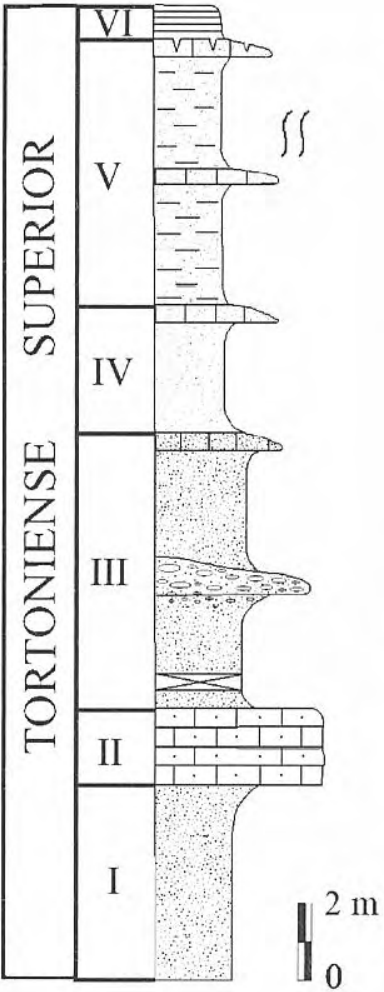

Banco de ostreidos

Margas

Arenas de grano fino

Arenas de grano medio

S: Conglomerados

Tramo cubierto

Figura 2. Columna litoestratigráfica y distribución vertical de la fauna de Clypeaster en el yacimiento estudiado.

Lithostratigraphic profile and vertical distribution of Clypeaster fauna in the studied outcrop.

\section{SISTEMÁTICA}

La mayor parte del material estudiado se encuentra depositado en la colección del Museo de Geología de la Universidad de Sevilla, el resto se ha dejado en el afloramiento para su mejor observación. En general se trata de ejemplares en buen estado de conservación. Todos los parámetros medidos se relacionan en la Fig. 3 y se expresan en milímetros (Tablas 1 a 7).

ORDEN CLYPEASTEROIDA Agassiz, 1872

Familia Clypeasteridae Agassiz, 1835

Género Clypeaster Lamarck, 1801

\section{Clypeaster brevior Seguenza, 1879}

Figs. 4 a-c

\begin{tabular}{|c|c|c|c|c|}
\hline & MGUS 2020 & Checchia & I. Smedile & Montenat \\
\hline L & 190 & 125 & 123 & 128 \\
\hline A & 167 & 113 & 111 & 110 \\
\hline h & 95 & 52 & 60 & 50 \\
\hline ma & 25 & - & - & - \\
\hline mp & 25 & - & - & - \\
\hline Lpp & 95 & - & - & - \\
\hline App & 53 & - & - & - \\
\hline Lpa & 89 & - & - & - \\
\hline Apa & 51 & - & - & - \\
\hline Lpi & 89 & - & - & - \\
\hline Api & 50 & - & - & - \\
\hline spm & 82 & - & - & - \\
\hline sp & 8 & - & - & - \\
\hline dp & 7 & - & - & - \\
\hline dpm & 24 & - & - & - \\
\hline
\end{tabular}

Tabla 1. Medidas en mm de C. brevior (MGUS 2020 ) procedente de Villanueva del Río y Minas comparadas con las dadas por Checchia-Rispoli (1925), Imbesi Smedile (1958) y Montenat y Roman (1970) para esta misma especie.

Measurements in mm of C. brevior (MGUS 2020) from Villanueva del Río y Minas in comparison with those given by Checcia-Rispoli (1925), Imbesi Smedile (1958) and Montenat and Roman (1970) for the same species.

1879 Clypeaster piramidalis var. brevior Seguenza, 66, lám. 9, fig. 1-5.

1913 Clypeaster altus (Klein) var. alticostatus (Michelin); Cottreau, 147,149, lám. 9, fig. 5.

1925 Clypeaster brevior; Checchia-Rispoli, 35, lám. 3, fig. 2; lám. 19, figs. 2, 2 a, 2 b.

1958 Clypeaster brevior; Imbesi Smedile, 26-27, lám. 7, figs. 2, a-c; 3, 4.

1970 Clypeaster aff. Brevior; Montenat y Roman, 110-111.

Material: Un ejemplar en buen estado de conservación (MGUS 2020), procedente del tramo IV.

\section{Descripción}

Especie de tamaño grande (máximo para esta especie: $190 \times 167 \times 95$ mm, Tabla 1), con el contorno pentagonal, ligeramente alargado y levemente sinuoso. Cara aboral de forma piramidal, esbelta en la zona del pétalo, con margen extenso, menos inclinado y saliente en la zona del pétalo, particularmente en los impares. Pétalos salientes y claviformes. En la zona porífera hay siete tubérculos como máximo en las placas. Espacio interzonal ancho, siendo tres veces la zona porífera. Interambulacro levemente saliente del margen al ápice. Madreporito poco deprimido entre la cresta y el pétalo. Ornamentación de la placa constituida por tuberculos de $0,5 \mathrm{~mm}$ de diámetro, separados entre sí por igual distancia, siendo esta menor en la parte alta del pétalo y mayor en la parte alta de la zona interzonal. Cara oral 

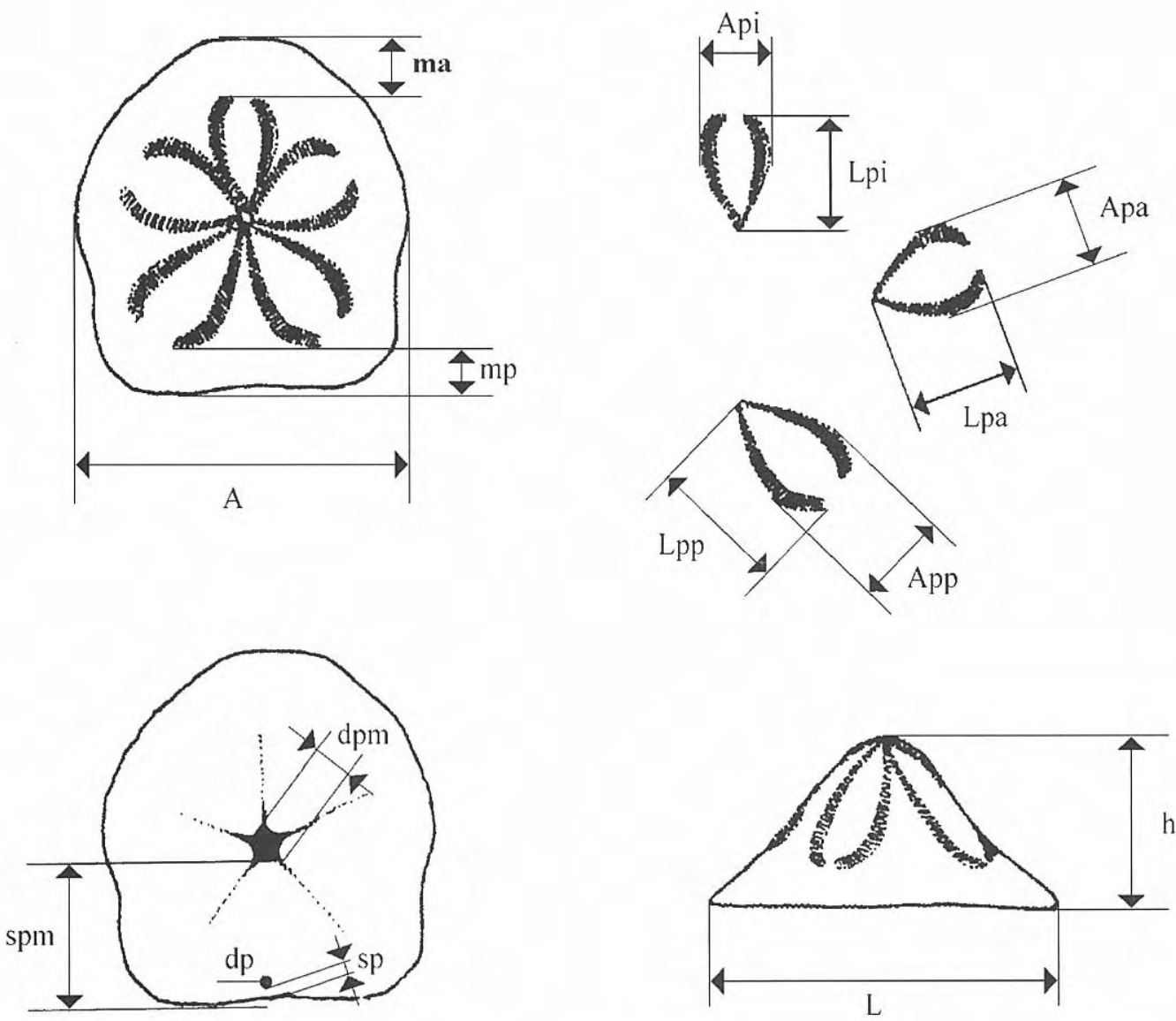

Figura 3. Principales caracteres morfológicos del género Clypeaster sobre los que se basan las descripciones taxonómicas del presente trabajo.

$\mathrm{L}=$ longitud; $\mathrm{A}=$ anchura $\mathrm{h}=$ altura; $\mathrm{ma}=$ margen anterior; $\mathrm{mp}=$ margen posterior; $\mathrm{Lpp}=$ longitud de los pétalos posteriores; App = anchura máxima de los pétalos posteriores; Lpa = longitud de los pétalos anteriores; Apa $=$ anchura máxima de los pétalos anteriores; Lpi = longitud del pétalo impar; Api = anchura máxima del pétalo impar; $\mathrm{spm}=$ separación del peristoma del borde posterior; $\mathrm{sp}$ = separación del periprocto del borde posterior; $\mathrm{dp}=$ diámetro del periprocto; dpm = diámetro del peristoma.

Main morphological characters of the genus Clypeaster used in the taxonomic description of the present paper.

$L=$ length $; A=$ width $; h=$ height; $m a=$ anterior margin; $m p=$ posterior margin Lpp = posterior paired petals length $; A p p=$ maximum posterior paired petals width $;$ Lpa $=$ anterior paired petals length Apa $=$ maximum anterior paired petals width; $\mathrm{Lpi}=$ anterior petal length; $\mathrm{Api}=$ maximum anterior petal width; spm = separation of the peristome from the posterior end; $s p=$ separation of the periproct fom the posterior border; $d p=$ periproct diameter; dpm = peristome diameter.

plana en cuatro quintas partes de la longitud, pero fuertemente inclinada hacia un profundo infundíbulo que termina en un peristoma pentagonal. Periprocto subcircular próximo al borde.

\section{Discusión}

La forma de esta especie se asemeja a la de $C$. portentosus Desmoulins aunque la cara apical en $C$. brevior es más aguda; la zona porífera en los dos tercios de su parte superior es rectilínea y convergente hacia el sistema apical (de figura falciforme), mientras que en $C$. portentosus es de forma más arqueada o de media luna.

\section{Distribución}

Según Imbesi Smedile (1958): Helveciense de Austria y
Calabria (Italia). Helveciense de Malta y Creta (Marcopoulou-Diacantoni, 1967). En España se ha citado en el Tortoniense de Murcia (Montenat y Roman, 1970).

\section{Clypeaster franchii Checchia-Rispoli, 1925}

Figs. 4 d-f

1925 Clypeaster franchii Checchia- Rispoli, 43, lám. 18, figs. 1 a,b.

1958 Clypeaster franchii; Imbesi Smedile, 37, lám.16, fig 2,a.b.

Material: Dos ejemplares juveniles en un estado de conservación mediocre (MGUS 2006, MGUS 2009), procedentes del tramo IV. 


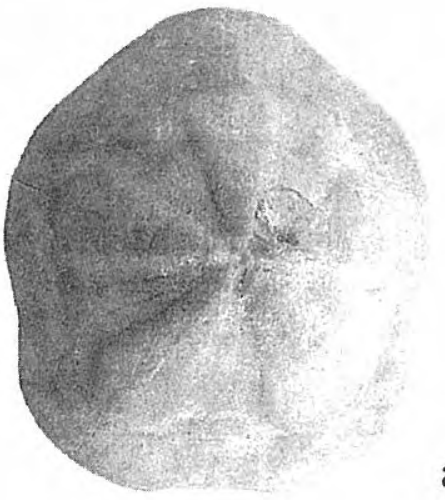

a

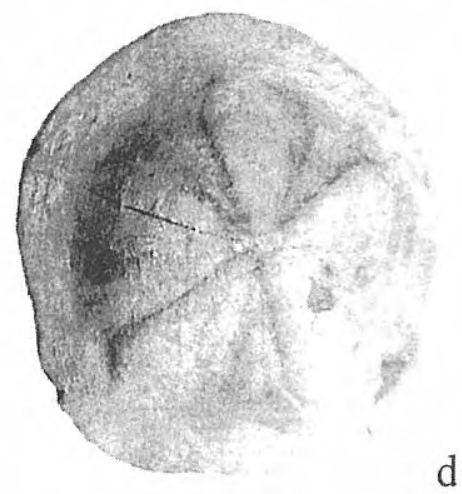

d

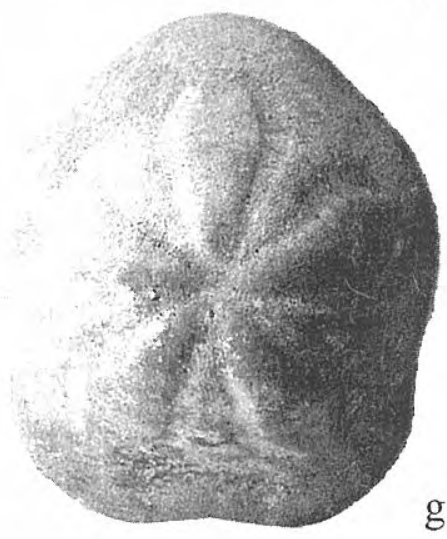

g

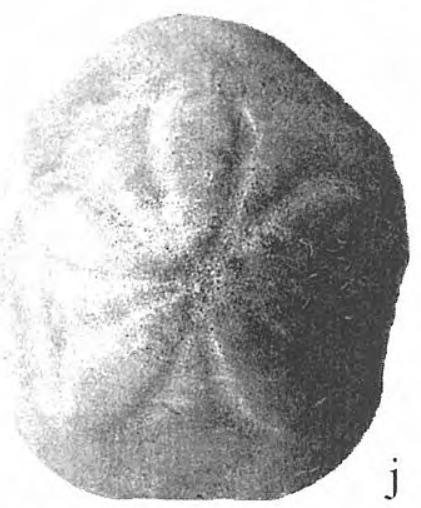

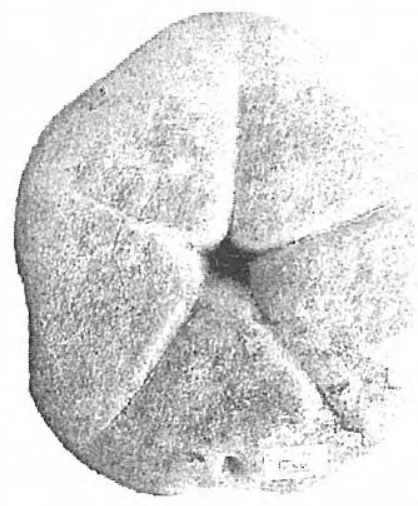

b
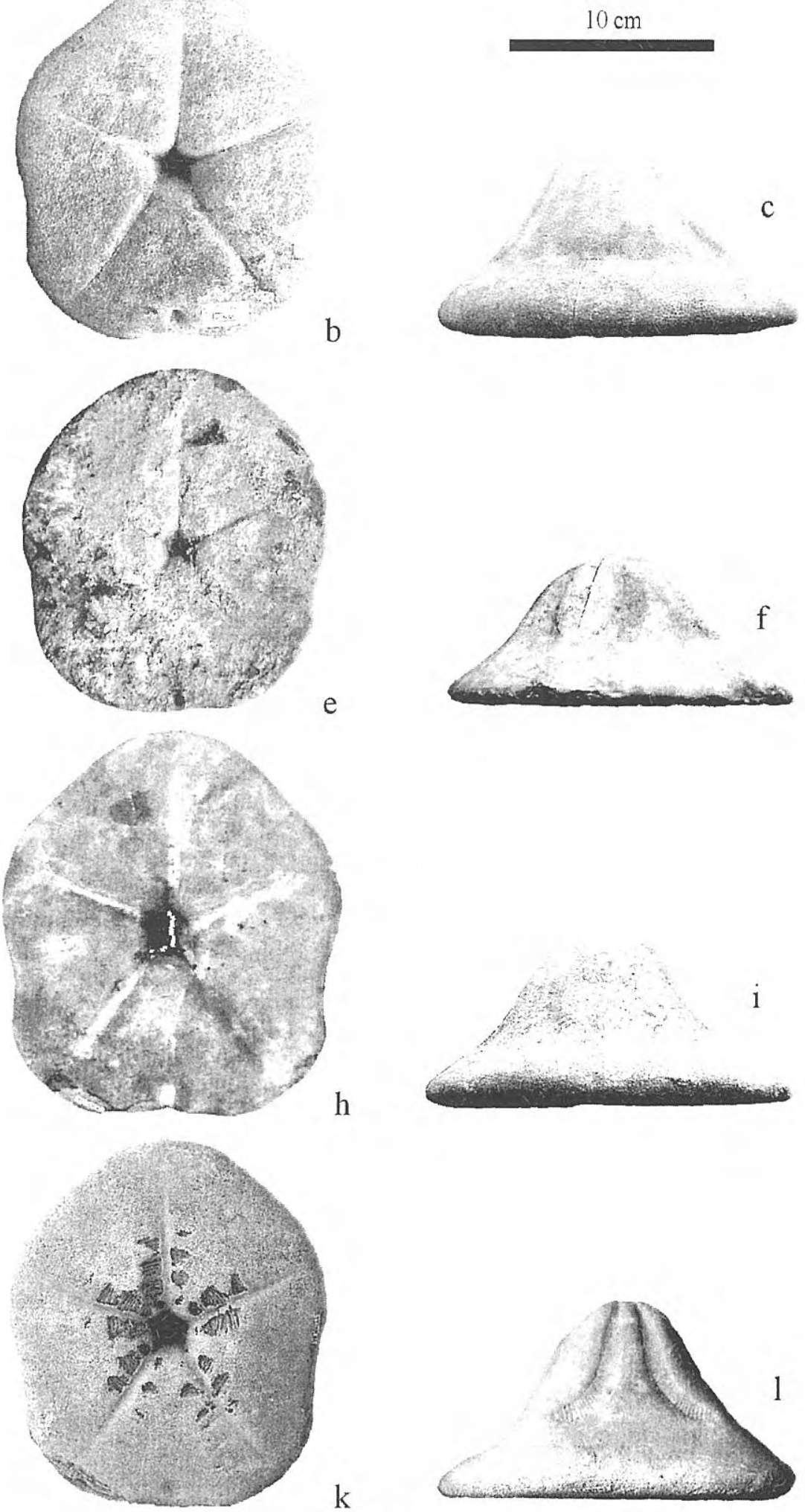

Figura 4.a-c. Clypeaster brevior Seguenza; a, vista aboral; b, vista oral; c, vista del perfil longitudinal; MGUS 2020, x 0,25. d-f. Clypeaster franchii Checchia-Rispoli; d, vista apical; e, vista oral; f, vista del perfil longitudinal; MGUS 2006, x 0,25. g-i. Clypeaster cermenatii Checchia-Rispoli; g, vista apical; h, vista oral; i, vista del perfil longitudinal; MGUS 2020, x 0,25. jI Clypeaster portentosus Desmoulins; j, vista apical; k, vista aboral; 1, vista del perfil longitudinal; MGUS 2003, x 0,25.

a-c. Clypeaster brevior Seguenza; a, aboral view; b, oral view; c, longitudinal profile view; MGUS 2020, x 0,25. $d$-f. Clypeaster franchii Checchia-Rispoli; d, apical view; e, oral view; f, longitudinal profile view; MGUS 2006, x 0,25. g-i. Clypeaster cermenatii Checchia-Rispoli; $g$, apical view; $h$, oral view; $i$, longitudinal profile view; MGUS 2020, x 0,25. $j$ l. Clypeaster portentosus Desmoulins; ; apical view; $k$, oral view; l, longitudinal profile view; MGUS 2003, x 0,25. 


\begin{tabular}{|c|c|c|c|}
\hline & MGUS 2006 & MGUS 2009 & Checchia \\
\hline L & 165 & 170 & 136 \\
\hline A & 153 & 160 & 130 \\
\hline h & 70 & 75 & 63 \\
\hline ma & 24 & 27 & - \\
\hline mp & 24 & 27 & - \\
\hline Lpp & 75 & 75 & - \\
\hline App & 44 & 40 & - \\
\hline Lpa & 74 & 72 & - \\
\hline Apa & 42 & 38 & - \\
\hline Lpi & 72 & 75 & - \\
\hline Api & 40 & 36 & - \\
\hline spm & 74 & 72 & - \\
\hline sp & - & 17 & - \\
\hline dp & - & - & - \\
\hline dpm & 13 & - & - \\
\hline
\end{tabular}

Tabla 2. Medidas en mm de C. franchii (MGUS 2006, MGUS 2009 ) de Villanueva del Río y Minas comparadas con las dadas por Checchia-Rispoli (1925) para esta misma especie.

Measurements in mm of C. franchii (MGUS 2006, MGUS 2009 ) from Villanueva del Río y Minas in comparison with those given by Checcia-Rispoli (1925) for the same species.

\section{Descripción}

Clypeaster de tamaño medio (con unas medidas máximas para esta especie de $170 \times 160 \times 75 \mathrm{~mm}$, Tabla 2), un poco más largo que ancho, con el contorno un poco circular, no sinuoso. Cara aboral elevada, siendo la región del pétalo cupuliforme y con zona marginal mucho menos inclinada que la primera, que es extensa y terminada en un borde grueso agudo en la parte posterior. Cara oral plana en gran parte, un poco inclinada hacia el estrecho infundíbulo. Sistema apical central. Pétalos un poco salientes, más anchos en el centro y cerrados hacia la extremidad. Estos iguales y largos: un poco más de dos tercios del radio. Zona porífera estrecha y poco deprimida. Espacio interzonal tres veces más ancho que la zona porífera, débilmente convexo en la región transversal, más saliente en la base que en la parte alta. Interambulacro ligeramente hinchado y un poco menos alto en el espacio interzonal. Peristoma pequeño, subcircular, situado en un infundíbulo estrechísimo y un poco más ancho en la parte externa. Periprocto pequeño, subcircular, situado a una distancia un poco mayor que su diámetro.

\section{Discusión}

La forma del contorno y del pétalo se aproxima mucho a las de $C$. philipii (Seguenza), pero su perfil es totalmente diferente a éste ya que es cónico y regular. $C$. franchii es ampliamente convexo en la parte alta, pero cóncavo en el flanco, especialmente en la parte anterior hacia la base del pétalo, siendo en la región marginal mucho menos inclinado.

\begin{tabular}{|c|c|c|c|}
\hline & MGUS 2002 & MGUS 2021 & Checchia \\
\hline L & 190 & 170 & 173 \\
\hline A & 165 & 150 & 150 \\
\hline h & 87 & 80 & 73 \\
\hline ma & 25 & 23 & - \\
\hline mp & 30 & 27 & - \\
\hline Lpp & 90 & 82 & - \\
\hline App & 46 & 40 & - \\
\hline Lpa & 85 & 80 & - \\
\hline Apa & 46 & 39 & - \\
\hline Lpi & 90 & 82 & - \\
\hline Api & 43 & 39 & - \\
\hline spm & 83 & - & - \\
\hline sp & 7 & - & - \\
\hline dp & 7 & - & - \\
\hline dpm & 25 & - & - \\
\hline
\end{tabular}

Tabla 3. Medidas en mm de C. cermenatii (MGUS 2002, MGUS 2021) de Villanueva del Río y Minas comparadas con las dadas por Checchia-Rispoli (1925).

Measurements in $\mathrm{mm}$ of $\mathrm{C}$. cermenatii (MGUS 2002, MGUS 2021) from Villanueva de Río y Minas in comparison with those given by ChecciaRispoli (1925).

\section{Distribución}

La especie proviene del Tortoniense de las localidades de Conidoni y Cessaniti (Calabria, sur de Italia). Se cita por vez primera fuera de esa región y amplía su distribución unos 2000 km al Oeste, confirmándose su edad: Tortoniense superior.

\section{Clypeaster cermenatii Checchia-Rispoli, 1925} Figs. $4 \mathrm{~g}-\mathrm{i}$

1925 Clypeaster cermenatii Checchia-Rispoli, 29, lám. 5, fig. 1.

Material: Dos ejemplares; el primero (MGUS 2002) en buen estado de conservación y el otro (MGUS 2021), con evidencias de bioerosión. Ambos proceden del tramo IV.

\section{Descripción}

Clypeaster de grandes dimensiones (máximas para esta especie de $190 \times 165 \times 87 \mathrm{~mm}$, Tabla 3), más largo que ancho, con contorno subdecagonal, y esquinas redondeadas. Cara aboral elevada, gibosa, fuertemente inclinada en la región del pétalo, ligeramente convexa a la mitad, con la mayor altura detrás del aparato apical. Región marginal extensa inclinada como la del pétalo, ligeramente prolongada detrás, terminada en un borde redondeado y un poco más agudizado en la parte trasera. Cara oral con la parte plana limitada sólo al margen, más inclinada en dirección al ancho infundíbulo. Sistema apical central. Pétalo largo, estrecho, un poco ovalado, saliente, un poco 


\begin{tabular}{|c|c|c|c|c|c|c|c|c|c|c|}
\hline & MGUS 2003 & MGUS 2004 & MGUS 2022 & MGUS 2024 & Michelin & Lovisato & Cottreau & Checc. & Smed. & Mont. \\
\hline L & 185 & 195 & 205 & 167 & 136 & 148 & 171 & 162 & 160 & 177 \\
\hline A & 162 & 160 & 165 & 152 & 128 & 135 & 151 & 150 & 140 & 150 \\
\hline h & 95 & 105 & 95 & 80 & 85 & 70 & 102 & 105 & 109 & 99,5 \\
\hline $\mathrm{ma}$ & 32 & 21 & 28 & 26 & - & - & - & - & - & - \\
\hline $\mathrm{mp}$ & 30 & 25 & 27 & 23 & - & - & - & - & - & - \\
\hline Lpp & 90 & 111 & 97 & 80 & - & - & - & 80 & - & - \\
\hline App & 38 & 42 & 50 & 43 & - & - & - & - & - & - \\
\hline Lpa & 87 & 97 & 91 & 78 & - & - & - & 80 & - & - \\
\hline Apa & 39 & 42 & 48 & 39 & - & - & - & - & - & - \\
\hline Lpi & 87 & 103 & 100 & 78 & - & - & - & - & - & - \\
\hline Api & 37 & 38 & 44 & 39 & - & - & - & - & - & - \\
\hline spm & 80 & 82 & 84 & 6 & - & - & - & - & - & - \\
\hline sp & 6 & 7 & 8 & - & - & - & - & 4 & - & - \\
\hline dp & 10 & 7 & 8 & - & - & - & - & - & - & - \\
\hline $\mathrm{dpm}$ & 22 & 22 & 25 & - & - & - & - & - & - & - \\
\hline
\end{tabular}

Tabla 4. Medidas en mm de C. portentosus (MGUS 2003, MGUS 2004, MGUS 2022, MGUS 2024) de Villanueva del Río y Minas comparadas con las dadas por Michelin (1861), Lovisato (1911), Cottreau (1913), Checchia-Rispoli (1925), Imbesi Smedile (1958) y Montenat y Roman (1970) para esta misma especie.

Measurements in mm of C. portentosus (MGUS 2003, MGUS 2004, MGUS 2022, MGUS 2024) from Villanueva del Rio y Minas in comparison with those given by Michelin (1861), Lovisato (1911), Cottreau (1913), Checchia-Rispoli (1925), Imbesi Smedile (1958) and Montenat and Roman (1970) for the same species.

arqueado en la parte alta y un poco redondeado en la base, donde son abiertos. Los pétalos impares dactiliformes, más estrechos y largos, extendiéndose cerca de tres cuartos del radio. El par anterior es más corto y más ancho. Zona porífera ancha, en la que la perteneciente a los pétalos impares se dispone perpendicularmente sobre el caparazón, por lo que presentan mayor apertura que los otros pares. En la extremidad son más arqueados cuanto más cerrados estén los pétalos. La zona porífera posterior de los pétalos pares es más flexuosa que la anterior. Espacio interzonal fuertemente convexo y estrecho en sección transversal. Interambulacro deprimido, siéndolo más en los anteriores que en los pares posteriores, los cuales en la línea mediana son sensiblemente abultados. Peristoma pequeño, pentagonal, situado en un infundíbulo no muy profundo. Surco ambulacral ancho, poco profundo, más ancho en dirección hacia el infundíbulo. Periprocto pequeño, circular, situado en el borde.

\section{Distribución}

La especie proviene del Tortoniense de la localidad de Belcastro (Calabria, sur de Italia). Se cita por vez primera fuera de esa localidad y se amplía su distribución unos 2000 $\mathrm{Km}$ al Oeste, confirmándose su edad: Tortoniense superior.

\section{Clypeaster portentosus Desmoulins, 1837} Figs. $4 \mathrm{j}-1$

1837 Clypeaster portentosus Desmoulins, 218.

1861 Clypeaster portentosus; Michelin, 125, lám. 28, figs. a-e.

1910 Clypeaster gustavi; Lovisato, 140, lám.16, figs. 1a-d.

1911 Clypeaster gauthieri; Lovisato, 37, lám. 4, figs. 1 a-d.
1913 Clypeaster altus (Klein) var. portentosus Cottreau, 45, lám. 7, figs. 1-4.

1914a Clypeaster canali; Lovisato: 4, lám. 1, figs. 2 a-d.

1914b Clypeaster griolii; Lovisato, 8, lám. 1, figs. 6 a -d.

1925 Clypeaster portentosus; Checchia- Rispoli, 35, lám. 3, figs. 1,1 a, 2, 5; lám 6 .

1958 Clypeaster portentosus; Imbesi Smedile, 21, lám. 4, fig. 2 a-b ; lám. 5, figs. 1,1 a, 2,2 a.

1970 Clypeaster portentosus; Mitrovic-Petrovic, lám. anexa a pág. 342.

1970 Clypeaster gustavi; Mitrovic-Petrovic, lám. anexa a pág 342.

1970 Clypeaster portentosus; Montenat y Roman, 106.

1972 Clypeaster portentosus; Comaschi Caria, 34, lám. 36, figs 1-3; lám 37, figs 1-3.

Material: Cuatro ejemplares adultos en buen estado de conservación (MGUS 2003, MGUS 2004, MGUS 2022, MGUS 2024); el primero procede del tramo V y los restantes del tramo IV.

\section{Descripción}

Clypeaster de muy notables dimensiones (máximas para esta especie de $205 \times 165 \times 95 \mathrm{~mm}$, Tabla 4), con esquinas redondeadas y de contorno un poco circular y lado sinuoso, correspondiendo la mayor anchura a la de los ambulacros pares. Cara aboral muy elevada, la parte superior es estrecha, cilíndrico-cónica y un poco inclinada delante; la parte inferior se ensancha bruscamente y desciende hacia un borde un poco menos inclinado. El perfil de la parte posterior es más inclinado que el anterior, así como más convexo. La parte alta de la pirámide está ligeramente doblada. Margen 


\begin{tabular}{|c|c|c|}
\hline & MGUS 2013 & Checchia \\
\hline $\mathrm{L}$ & 143 & 140 \\
\hline $\mathrm{A}$ & 125 & 120 \\
\hline $\mathrm{h}$ & 55 & 50 \\
\hline $\mathrm{ma}$ & 14 & - \\
\hline $\mathrm{mp}$ & 16 & - \\
\hline $\mathrm{Lpp}$ & 65 & - \\
\hline $\mathrm{App}$ & 35 & - \\
\hline $\mathrm{Lpa}$ & 65 & - \\
\hline Apa & 34 & - \\
\hline Lpi & 64 & - \\
\hline Api & 32 & - \\
\hline $\mathrm{spm}$ & 66 & - \\
\hline $\mathrm{sp}$ & 4 & - \\
\hline $\mathrm{dp}$ & 6 & - \\
\hline $\mathrm{dpm}$ & 10 & - \\
\hline
\end{tabular}

Tabla 5. Medidas en mm de C. aichinoi (MGUS 2013 ) de Villanueva del Río y Minas comparadas con las dadas por Checchia-Rispoli (1925) para esta misma especie.

Measurements in mm of C. aichinoi (MGUS 2013) from Villanueva del Río y Minas in comparison with those given by Checcia-Rispoli (1925) for the same species.

corto, terminado en un borde redondeado y agudo que permanece constante, excepto en las proximidades del periprocto. Cara oral extensamente plana inclinándose en la dirección del infundíbulo. Sistema apical central, situado en una ligera depresión de la cresta del pétalo. Madreporito pequeño, en forma de estrella, de pequeño diámetro. Poros genitales pequeños, circulares, a distinta distancia del madreporito, el más lejano a unos tres milímetros de este. Pétalos muy largos, sobresalientes, estrechos, un poco arqueados en la parte alta y casi rectos en la base; la zona porífera es subparalela en la zona más saliente pero luego en la extremidad inferior se ajusta para cerrar el pétalo. Todos los pétalos tienen la misma longitud a excepción de los posteriores, que son un poco más largos debido a la inclinación de la pirámide hacia delante. Zona porífera ancha, situada en la parte lateral del pétalo, un poco lisa en la base. Espacio interzonal ancho, sobresaliente, fuertemente convexo en la sección transversal, siendo más sobresaliente hacia la mitad del pétalo y más deprimido en la base y en la parte alta. Interambulacro longitudinalmente serrado tras la zona porífera, estrecho y ensanchado suavemente hacia la parte distal. Peristoma pequeño, subcircular, situado en un infundíbulo profundo, bien desarrollado con una pared subvertical, más amplio hacia el borde que se hunde profundamente en un ancho surco ambulacral, que se atenúa bruscamente en la región marginal. Periprocto pequeño, circular, distante del margen apenas cuatro milímetros. Tubérculos pequeños y muy distantes en la cara apical: en el espacio interporífero están dispuestos en tres series en cada placa, un poco más grandes y densos hacia el borde y en el interambulacro de la cara aboral.

\section{Discusión}

Clypeaster portentosus es uno de los Clypeaster más polimorfos, tal y como señaló Imbesi Smedile (1958), quien estableció cinco morfotipos diferentes para esta especie. A primera vista puede confundirse con Clypeaster altus, aunque éste no tiene ni la forma ni el margen, ni los pétalos que tiene $C$. portentosus, ya que $C$. altus tiene una forma netamente pentagonal, acampanada en la parte alta, con borde menos sinuoso, pétalo más corto y abierto, con el borde redondeado y un perfil más convexo que $C$. portentosus.

\section{Distribución}

Esta especie se ha citado en el Helveciense de Calabria y de Cagliari (Italia), Tortoniense de Yugoslavia y Marruecos; en el Mioceno: Argelia, Pianosa (Italia), Córcega y Malta; en el Messiniense de Marruecos. En España se ha citado en el Mioceno de las Islas Baleares (Mallada, 1892; Lambert 1928) y en el Tortoniense de Alicante (Montenat y Roman, 1970).

\section{Clypeaster aichinoi Checchia-Rispoli, 1925} Figs. 5 a-c

1925 Clypeaster aichinoi Checcia-Rispoli, 30, lám. 8, fig 2; lám. 14, fig. 3.

Material: Un ejemplar en buen estado de conservación (MGUS 2013), procedente del tramo IV.

\section{Descripción}

Clypeaster de dimensiones medias (máxima para esta especie de 143 x 123 x 55 mm, Tabla 5), más largo que ancho, con contorno pentagonal, y esquinas redondeadas, con la anterior más saliente que las otras y lado sinuoso, especialmente el posterior. Cara aboral elevada, gibosa, redondeada en la parte alta, y perfil antero-posterior muy en declive, convexo y rectilíneo delante. Margen estrecho, con la misma inclinación del casquete del pétalo, grueso cerca del pétalo y terminado en un borde muy agudo. Cara oral casi plana cerca de la mitad de su extensión, para inclinarse rápidamente en un larguísimo infundíbulo. Pétalos salientes, iguales, ovalados, abiertos en la extremidad, los impares más sobresalientes y casi dactiliformes. Zona porífera ancha, situada en la parte lateral del pétalo, un poco más aplanada que los pétalos pares. Espacio interzonal ancho, sobresaliente, un poco aplanado en la zona mediana; el pétalo continúa siendo sobresaliente por toda el área ambulacral, pero no así en el margen. Interambulacro deprimido y más ancho en la base de la zona porífera, especialmente el posterior. La depresión no se manifiesta en el borde, donde es más suavizada. Peristoma grande, pentagonal, situado en un profundo y larguísimo infundíbulo con una pared vertical, un poco más amplio hacia el borde. Canal ambulacral ancho y profundo. Periprocto pequeño, circular, situado muy al borde.

\section{Discusión}

Este Clypeaster tiene un perfil casi idéntico al de Clypeaster olisiponensis (Michelin), pero este último 


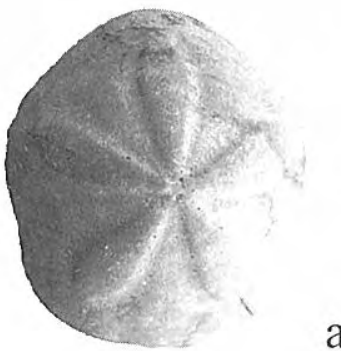

a
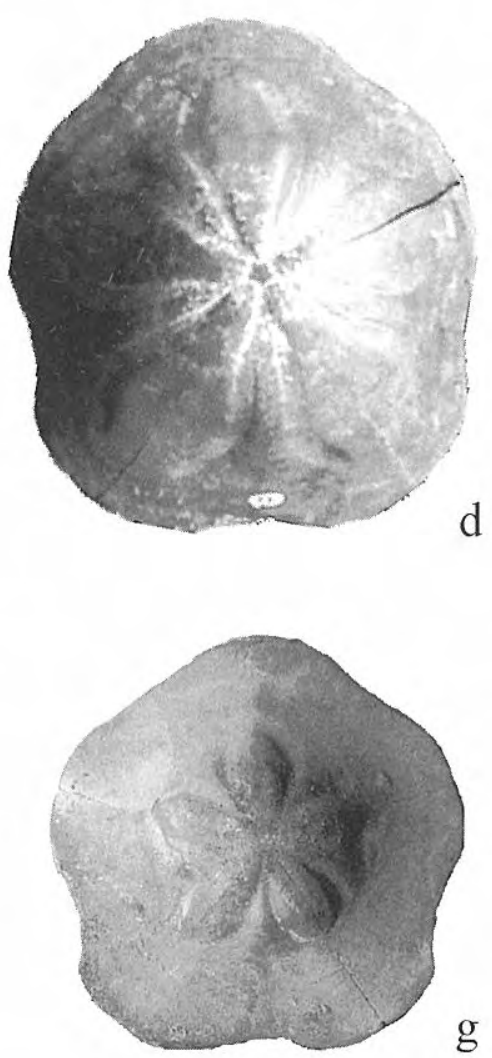

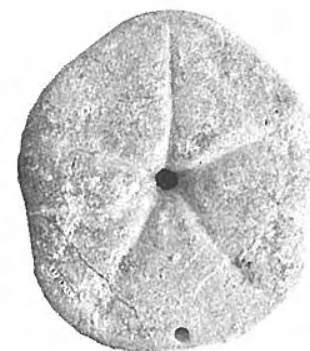

b
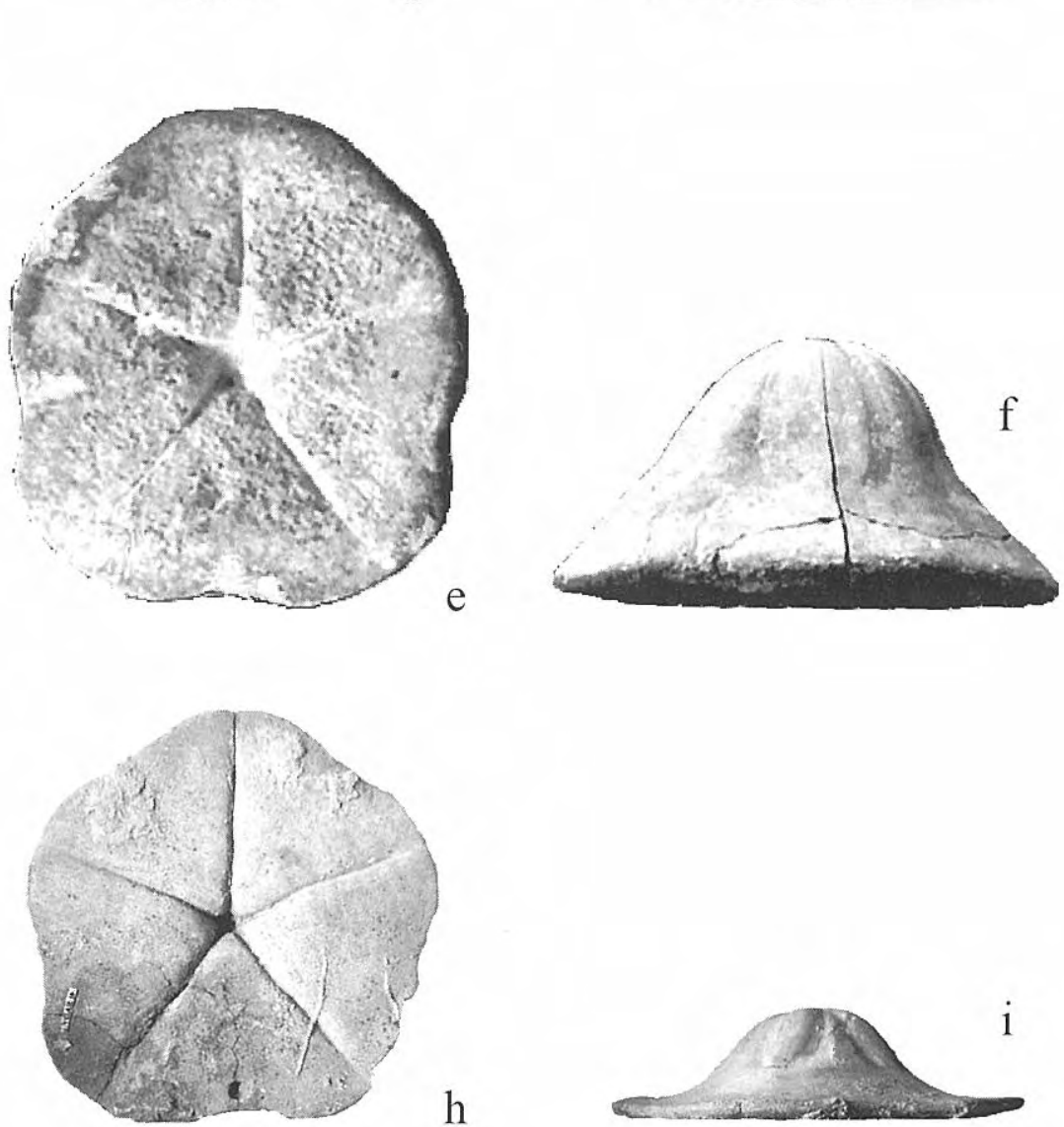

$10 \mathrm{~cm}$

c

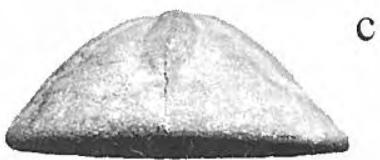

b

h

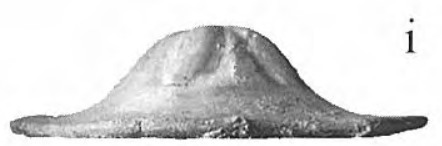

Figura 5. a-c. Clypeaster aichinoi Checchia-Rispoli; a, vista aboral; b, vista oral; c, vista del perfil longitudinal; MGUS 2013, x 0,25. d-f. Clypeaster tauricus Desor; d, vista aboral; e, vista oral; f, vista del perfil longitudinal; MGUS 2023, $\mathrm{x}$ 0,25. g-i. Clypeaster sp.; g, vista apical; h, vista oral; i, vista del perfil longitudinal; MGUS 2010, x 0,25.

a-c. Clypeaster aichinoi Checchia-Rispoli; $a$, aboral view; $b$, oral view; , longitudinal profile view; MGUS $2013, x$ 0,25. d-f. Clypeaster tauricus Desor; $d$, aboral view; e, oral view; $f$, longitudinal profile view; MGUS $2006, x 0,25$. g-i. Clypeaster sp.; g, aboral view; h, oral view; $i$, longitudinal profile view; MGUS 2010, x 0,25.

tiene el borde más agudo y más sinuoso, el pétalo más largo y más abierto, el interambulacro más deprimido, el peristoma más grande y el infundíbulo menos amplio pero más profundo.

\section{Distribución}

La especie proviene del Tortoniense de la localidad de Belcastro (Calabria, sur de Italia). Se cita por primera vez fuera de esa localidad y se amplía su distribución unos 2000 $\mathrm{Km}$ al Oeste, confirmándose su edad: Tortoniense superior.
1861 Clypeaster tauricus; Michelin, 108, lám 10, 11.

1866 Clypeaster tauricus; Fischer, 207, lám. 18, fig. 1.

1913 Clypeaster altus var. Tauricus; Cottreau, 145-149, lám. 9, fig. 8.

1952 Clypeaster tauricus; Pinar, 22, lám. 1, fig. 5.

1958 Clypeaster tauricus; Imbesi Smedile, 34, lám. 13, fig 2a-b.

1960 Clypeaster tauricus; Roman, 78, lám. 3, fig. 5.

Material: Un ejemplar adulto presentando buen estado de conservación (MGUS 2023), procedente del tramo IV.

\section{Descripción}

Clypeaster de grandes dimensiones (Tabla 6), con el 


\begin{tabular}{|c|c|c|c|c|}
\hline & MGUS 2023 & Fischer & I. Smedile & Roman \\
\hline L & 217 & 190 & 182 & 145 \\
\hline A & 192 & 160 & 157 & 121 \\
\hline h & 108 & 65 & 65 & 54 \\
\hline ma & 30 & - & - & - \\
\hline mp & 26 & - & - & - \\
\hline Lpp & 107 & - & - & - \\
\hline App & 62 & - & - & - \\
\hline Lpa & 97 & - & - & - \\
\hline Apa & 58 & - & - & - \\
\hline Lpi & 103 & - & - & - \\
\hline Api & 50 & - & - & - \\
\hline spm & 97 & - & - & - \\
\hline sp & 10 & - & - & - \\
\hline dp & 6 & - & - & - \\
\hline dpm & 24 & - & - & - \\
\hline
\end{tabular}

Tabla 6. Medidas en mm de C. tauricus (MGUS 2023) de Villanueva del Río y Minas comparadas con las dadas por Fischer (1866), Imbesi Smedile (1958) y Roman (1960) para esta misma especie.

Measurements in mm of C. tauricus (MGUS 2023) of Villanueva del Río y Minas in comparison with those given by Fischer (1866), Imbesi Smedile (1958) and Roman (1960) for the same specie.

contorno pentagonal, alargado y marcadamente sinuoso. La cara aboral se caracteriza por las dimensiones sobresalientes de los pétalos y por la exigua depresión existente entre estos. En la zona interambulacral, la zona porífera resulta poco inclinada, lo que deriva en una forma general acampanada, alargada anteroposteriormente, con el ápice desplazado hacia el lado posterior. Margen relativamente poco extenso, borde muy redondeado en la parte anterior, aunque menos en la parte posterior. Pétalo claviforme, muy ensanchado hacia la extremidad distal, poco saliente; zona porífera muy ancha, poco deprimida. Interambulacro poco saliente. Tubérculos de $0,6 \mathrm{~mm}$, distanciados nueve $\mathrm{mm}$ en el borde. Cara oral plana, con un infundíbulo profundo y ancho. Peristoma subpentagonal. Periprocto subelíptico.

\section{Distribución}

Esta especie ha sido citada en el Neógeno de Creta, y en el Helveciense de Karaman (Turquía). Se cita por primera vez en España.

\section{Clypeaster sp.}

Figs. 5 g-i

Material : Dos ejemplares adultos en buen estado de conservación (MGUS 2010, MGUS 2016), procedentes del tramo V.

\section{Descripción}

Clypeaster de dimensión mediana (máxima de 180 x 175 x $50 \mathrm{~mm}$, Tabla 7), con contorno subdecagonal y esquinas redondeadas. Cara aboral elevada y gibosa, siendo la extensa

\begin{tabular}{|c|c|c|}
\hline & MGUS 2010 & MGUS 2016 \\
\hline L & 177 & 159 \\
\hline A & 177 & 160 \\
\hline $\mathrm{h}$ & 52 & 55 \\
\hline ma & 47 & 40 \\
\hline mp & 52 & 42 \\
\hline Lpp & 52 & 52 \\
\hline App & 27 & 28 \\
\hline Lpa & 51 & 47 \\
\hline Apa & 27 & 27 \\
\hline Lpi & 55 & 50 \\
\hline Api & 28 & 28 \\
\hline spm & - & 65 \\
\hline sp & 5 & 7 \\
\hline dp & 8 & 6 \\
\hline dpm & - & - \\
\hline
\end{tabular}

Tabla 7. Medidas en mm de Clypeaster sp. (MGUS 2010, MGUS 2016) de Villanueva del Río y Minas.

Measurements in $\mathrm{mm}$ of Clypeaster sp. (MGUS 2010, MGUS 2016) from Villanueva del Río y Minas.

región marginal delgada y ligeramente cóncava. Sistema apical central situado en una ligera depresión de las crestas de los pétalos, en forma de estrella. Pétalos cortos y de igual longitud, siendo sobresalientes, anchos y fusiformes. Zona porífera estrecha y deprimida. Espacio interzonal ancho y sobresaliente. Cara oral plana e inclinada sólo en las proximidades del estrecho infundíbulo. Peristoma pequeño, pentagonal, situado en un infundíbulo profundo. Periprocto grande, circular, situado en el borde.

\section{Discusión}

La forma de este Clypeaster se asemeja a la de $C$. marginatus, aunque este último es menos elevado en la cara apical y menos giboso, con los pétalos menos sobresalientes, siendo su margen más extenso (2/3 del radio) que en Clypeaster sp. (1/3 del radio). La cara oral de $C$. marginatus es plana solamente en el margen, inclinándose progresivamente hacia un ancho infundíbulo; sin embargo, en Clypeaster sp. es plana casi toda la cara oral, inclinándose tan sólo cerca del estrecho infundíbulo, por lo que pudiera tratarse de una nueva especie.

\section{CONCLUSIONES}

Se confirma la presencia de Clypeaster brevior, $C$. franchii, $C$. cermenatii, $C$. portentosus, $C$. aichinoi y $C$. tauricus, en las facies areno-limosas del Mioceno Superior en el sector noroccidental de la Cuenca del Guadalquivir (Villanueva del Río y Minas, provincia de Sevilla). Cinco de estas especies: $C$. franchii, $C$. cermenatii, C. aichinoi, C. tauricus y Clypeaster sp., son 
citadas por primera vez en la Península Ibérica, ampliándose su registro hacia el dominio Atlántico durante el Tortoniense.

Se instaló un ambiente litoral somero de aguas agitadas y substrato de arenas de grano medio y grueso (tramos III y IV), que correspondería a un episodio favorable para el desarrollo de Clypeaster de gran tamaño y bordes gruesos. La progresiva evolución hacia un medio menos energético y más profundo, con arenas finas y limosas (tramo V), pudo propiciar la desaparición de todas las especies excepto $C$. portentosus, y la aparición de otras con menor tamaño y borde más fino, como Clypeaster sp. Esta última especie probablemente sea endémica del Tortoniense del sector occidental de la Cuenca del Guadalquivir, aunque no se describe formalmente como una nueva especie dado que los especímenes encontrados hasta el momento son escasos.

\section{AGRADECIMIENTOS}

Mi más sincero agradecimiento al Dr. E. Mayoral (Dpto. de Geodinámica y Paleontología, Univ. de Huelva), y al Dr. J. Gallemí (Museo de Geología, Barcelona), por su ayuda y asesoramiento en la elaboración de este trabajo. A D. Rey (Barcelona) y al Dr. L. Cotroneo (Reggio Calabria, Italia) por su ayuda en la bibliografía. Asimismo deseo agradecer al Dr. R.H. Wagner (Jardín Botánico de.Córdoba) la realización de las fotografías y a J. Gil (Sevilla) por la ayuda en el trabajo de campo. Agradezco también el trabajo de los editores que ha contribuido a mejorar el texto original.

\section{BIBLIOGRAFÍA}

Boggild G. R. and Rose E.P.F. 1985. Echinoid biofacies as paleoenvironmental indices. Annales de géologie des Pays helléniques, 31 (2), 57-67.

Checchia-Rispoli, G. 1925. Illustrazione dei Clipeastri miocenici della Calabria. Seguita da uno studio sulla morfologia interna e sulla classificazione dei clipeastri. Memoria per servire alla descrizione della carta geologica d' Italia, 9, 1-75.

Comaschi Caria, I. 1972. Gli echinidi del Miocene della Sardegna. Stabilimento Tipografico Editoriale Fossataro, Cagliari, 1-95.

Cotteau J, 1890. Échinides éocènes de la province d'Alicante (Espagne). Mémoires de la Société Géologique de France, 3 (5), 1-107.

Cottreau, J. 1913. Les Echinides néogènes du basin méditerranéen. Annales de l'Institut océanographique, 6 (3), 1-193.

Desmoulins, C. 1837. Troisième Mémoire sur les Échinides. Synonymie générale. Actes Société Linnéenne Bordeaux, 9, 45-364.

Desor, E. 1853-1858. Synopsis des échinides fossiles. Reinwald, Paris \& Wisbaden, 490 pp.

Fischer, P. 1866. Asie mineure. Description physique de cette contrée, Paleontologie, Faune tertiaire moyenne. L. Guérin et Cie, Paris, 235 pp.
Galán, E., Hernández, Mª J., Mayoral, E., y Miras, A. 1993. Guía del Museo de Geología de la Universidad de Sevilla, Servicio de Publicaciones de la Universidad de Sevilla, 184 pp.

García, A., Pignatelli, R., Crespo, A., Torres, T., Martínez, C., Granados, L., del Pan, T., Argüelles, A., de las Eras, A. y Leiva, F. 1975. Mapa Geológico de España, escala 1:50.000, Explicación de la Hoja $N^{\circ}$ 963, Lora del Río. Instituto Geológico y Minero de España, 1-17.

Imbesi Smedile, M. 1958. Clypeastri aquitaniani, elveciani e tortoniani della Calabria. Paleontographia Italica, 53, 147.

Kilian, W. 1892. Estudio paleontológico acerca de los terrenos secundarios y terciarios de Andalucía. Boletín de la Comisión del Mapa Geológico, 19, 263-386.

Lamarck, J. B. 1801. Système des animaux sans vertèbres, ou tableu général des classes, des ordres et des genres de ces animaux. Paris, $432 \mathrm{pp}$.

Lambert J. 1928. Révision des échinides fossiles de la Catalogne. 2e partie: échinides du Pliocène et échinides néogènes des Baléares. Memorias del Museo de Ciencias Naturales de Barcelona, Serie Geológica, 1 (2), 1-62.

Lovisato, D. 1910. Una parola sul Clypeaster lovisatoi Cotteau e specie nuove di Clypeaster ed Echinolampas. Paleontographia Italica, Pisa, 16, 137-145.

Lovisato, D. 1911. Note di paleontologia miocenica della Sardegna. Specie nuove di Clypeaster e di Amphiope. Paleontographia Italica, Pisa, 17, 37-47.

Lovisato, D. 1914a. Fibularidi e clipeastridi miocenici della Sardegna. Bollettino della Società Geologica Italiana, Roma, 33, 485-564.

Lovisato, D. 1914b. Altre specie nuove di Clypeaster, Scutella ed Amphiope della Sardegna. Rivista Italiana di Paleontologia, Parma, 20, 89-121.

Mallada, L. 1892. Catálogo general de las especies fósiles encontradas en España. Boletín de la Comisión del Mapa Geológico de España, 18, 253 pp.

Marcopoulou-Diacantoni, A. 1967. I panis ton neogenon echinon ton ellinikon choron. Annales géologie des Pays helléniques. Atenas, 18, 331-406.

Michelin, H. 1861. Monographie des Clypéastres fossiles. Mémoires de la Société Géologique de France, 2 ème série, 7(2), 101-145.

Mitrovic-Petrovic, J. 1970. Les caractéristiques biostratigraphiques et paléoécologiques des échinides du Miocène en Yougoslavie. Comittee Mediterranean Neogene Stratigraphy, Giornale di geologia, Bologne, 35 (1), 335-346.

Montenat, C. et Roman, J. 1970. Échinides néogènes d'Espagne (Provinces d'Alicante et de Murcie) Annales de Paléontologie (Invertébrés), 56 (1), 87-138.

Pinar, N. 1952. Sur une méthode de détermination de Clypeaster d'Anatolie. Bulletin de la Faculté des Sciences de Istambul, 17 (1), 1-22.

Roman, J. 1960. Échinides (Clypeaster, Scutella, Shizaster) de Hèlvetien du bassin de Karaman (Turquie). Bulletin of the Mineral Researh and Exploration Institute of Turkey, Ankara, 55, 64-94.

Seguenza, G. 1879. Le formazioni terziarie nella Provincia 
di Reggio (Calabria). Atti Reale Accademia dei Lincei, Roma, 3 (6), 1-446.

Serres, M. de 1829. Géognosie des terrains tertiaires ou tableau des principaux animaux invertébrés des terrains marins tertiaires du Midi de la France. Pomathio-
Durville édit., Montpellier, 1-276.

Sierro, F.J., Civis, J., González Delgado, J.A., Flores, J.A. and Dabrio, C.J. 1990. The Neogene of the Guadalquivir basin (SW Spain). Paleontologia i Evolució, Memòria Especial, 2, 209-250.

Manuscrito recibido: 6 de marzo, 2001 Manuscrito aceptado: 27 de marzo, 2002 